\title{
ON A THEOREM OF R. JUNGEN
}

\section{P. SCHÜTZENBERGER}

Let us recall the following elementary result in the theory of analytic functions in one variable.

TheOREM ( $R$. Jungen [7]). If $a$ is rational and $b$ algebraic their Hadamard product $c$ is algebraic; if, further, $b$ is rational, $c$ also is rational.

For several variables, Jungen's proof shows that the theorem is still true for the Bochner-Martin [2] Hadamard product. It does not hold for the Cameron-Martin [3] and for the Haslam-Jones [6] Hadamard products. In this note we give a version of Jungen's theorem which is valid for a restricted interpretation of the notions involved when $a$ and $b$ are formal power series in a finite number of noncommuting variables.

1. Notations. Let $R$ be a fixed not necessarily commutative ring with unit 1 . For any finite set $Z, F(Z)$ is the free monoid generated by $Z$ and $R_{\text {pol }}(Z)$ is the free module on $F(Z)$ over $R$. An element $a$ of $R_{\mathrm{pol}}(Z)$ will usually be written in the form $a=\sum\{(a, f) \cdot f: f \in F(Z)\}$ where the coefficients $(a, f)$ are in $R ; R_{\text {pol }}(Z)$ is graded in the usual manner and $\pi_{n} a=\sum\{(a, f) \cdot f: f \in F(Z), \operatorname{deg} f \leqq n\}$. We identify $R$ with $\pi_{0} R_{\mathrm{pol}}(Z) . \quad R_{\mathrm{pol}}(Z)$ is also a ring with product $a a^{\prime}$ $=\sum\left\{\left(a, f^{\prime}\right)\left(a^{\prime}, f^{\prime \prime}\right) \cdot f: f, f^{\prime}, f^{\prime \prime} \in F(Z), f=f^{\prime} f^{\prime \prime}\right\}$.

It is well known (cf., e.g., $[4 ; 3])$ that these notions extend to the ring $R(Z)$ of the formal power series (with coefficients in $R$ ) in the noncommuting variables $z \in Z ; R(Z)$ is topologized in the same manner as a ring of commutative formal power-series and $a a^{\prime}$ $=\lim _{n, n^{\prime} \rightarrow \infty}\left(\pi_{n} a\right)\left(\pi_{n^{\prime}} a^{\prime}\right)$. Any $b \in R^{*}(Z)=\left\{a \in R(Z): \pi_{0} a=0\right\}$ has a quasi-inverse $(-b)^{*}=\lim _{n \rightarrow \infty} \sum_{n^{\prime}<n}(-b)^{n^{\prime}}$. If $a$ is invertible, $a^{-1}=\left(1+b^{*}\right)\left(\pi_{0} a^{-1}\right)$ where $b=-\left(\pi_{0} a^{-1}\right)\left(a-\pi_{0} a\right) \in R^{*}(Z)$. We shall say that $S^{*} \subset R^{*}(Z)$ is rationally closed if $r, r^{\prime} \in R, b, b^{\prime} \in S^{*}$ imply $r b+b^{\prime} r^{\prime}, b b^{\prime}, b^{*} \in S^{*}$. If this is so, the set of those elements $a$ of $R(Z)$ such that $a-\pi_{0} a \in S^{*}$ is a ring containing the inverses of its invertible elements.

Definition 1. $R_{\mathrm{rat}}^{*}(X)$ is the least rationally closed subset (of $R(X)$ ) containing $X$.

Now let $Y=\left\{y_{j}\right\}$ be a set of a finite number $M$ of new variables and $R^{M}(X \cup Y)$ (resp. $R_{\mathrm{pol}}^{M}(X \cup Y)$ ) the cartesian product of $M$ copies

Received by the editors December 6, 1961. 
of the $R$-module $R(X \cup Y)$ (resp. $R_{\mathrm{pol}}^{M}(X \cup Y)$ ). For each $q=\left(q_{1}, \cdots, q_{m}\right) \in R^{M}(X \cup Y), \quad \pi_{n} q=\left(\pi_{n} q_{1}, \cdots, \pi_{n} q_{m}\right) . \quad$ If $q \in R^{* M}(X \cup Y)$ (i.e., if $\left.\pi_{0} q=0\right)$ let $\lambda_{q}$ be the homomorphism of the monoid $F(X \cup Y)$ into the multiplicative monoid structure of $R(X \cup Y)$ that is induced by $\lambda_{q} x=x$ if $x \in X$ and $\lambda_{q} y_{j}=q_{j}$ if $y_{j} \in Y$. Since $\pi_{0} q=0, \lambda_{q}$ can be extended to an endomorphism of the $R$ module $R(X \cup Y)$ by $\lambda_{q} a=\sum\left\{(a, f) \lambda_{q} f: f \in F(X \cup Y)\right\}$; also, $\lambda_{q} p$ $=\left(\lambda_{q} p_{1}, \cdots, \lambda_{q} p_{M}\right)$ for any $p \in R^{M}(X \cup Y)$.

We shall say that $p \in R^{* M}(X \cup Y)$ is a proper system if $\left(p_{j}, \jmath_{j^{\prime}}\right)=0$ for all $j, j^{\prime} \leqq M$. Then, if $q \in R^{* M}(X), \lambda_{q} p \in R^{* M}(X)$ and $\pi_{n+1} \lambda_{q} p$ $=\pi_{n+1} \lambda_{\pi_{n} q} p$ for all $n$. Consider now the infinite sequence $p(0)=0$, $p(1)=\lambda_{p(0)} p, \cdots, p(m+1)=\lambda_{p(m)} p, \cdots$. Trivially, $\pi_{m^{\prime}} p\left(m^{\prime}\right)$ $=\pi_{m^{\prime}} p\left(m^{\prime}+m^{\prime \prime}\right) \in R^{* M}(X)$ for $m^{\prime}=0$ and all $m^{\prime \prime}$. If these relations hold for $m^{\prime} \leqq m$, they still hold for $m+1$ because

$$
\begin{aligned}
\pi_{m+1} p(m+1) & =\pi_{m+1} \lambda_{p(m)} p=\pi_{m+1} \lambda_{\pi_{m} p(m)} p=\pi_{m+1} \lambda_{\pi_{m} p\left(m+m^{\prime \prime}\right)} p \\
& =\pi_{m+1} \lambda_{p\left(m+m^{\prime \prime}\right)} p=\pi_{m+1} p\left(m+1+m^{\prime \prime}\right) .
\end{aligned}
$$

Hence, $p(\infty)=\lim _{m \rightarrow \infty} p(m)$ exists and it satisfies $p(\infty) \in R^{* M}(X)$, $\pi_{0} p(\infty)=0, p(\infty)=\lambda_{p(\infty)} p$. In fact, $p(\infty)$ is the only element to satisfy these equations because if $\pi_{0} p^{\prime}=0$ and $p^{\prime}=\lambda_{p^{\prime}} p$, any relation $\pi_{m} p(\infty)$ $=\pi_{m} p^{\prime}$ implies $\pi_{m+1} p^{\prime}=\pi_{m+1} \lambda_{\pi_{m} p^{\prime}} p=\pi_{m+1} \lambda_{\pi_{m} p(\infty)} p=\pi_{m+1} p(\infty)$. For this reason we call $p(\infty)$ the solution of $p$.

Definition 2. $R_{\mathrm{alg}}^{*}(X)$ is the least subset (of $R^{*}(X)$ ) that contains every coordinate of the solution of any proper system having its coordinates in $R_{\text {pol }}^{*}(X \cup Y)$.

(REMARK. It can easily be shown that $R_{\mathrm{alg}}^{*}(X)$ is rationally closed and that it contains every coordinate of the solution of any proper system having its coordinates in $R_{\text {alg }}^{*}(X \cup Y)$.)

Definition 3. For any

$$
a, b \in R(X), \quad a \odot b=\sum\{(a, f)(b, f) \cdot f: f \in F(X)\} .
$$

\section{Main result.}

Property 2.1. The element $a$ of $R^{*}(X)$ belongs to $R_{\mathrm{rat}}^{*}(X)$ if and only if there exists a finite integer $N \geqq 2$ and a homomorphism $\mu$ of $F(X)$ into the multiplicative monoid of $R^{N \times N}$ (the ring of the $N \times N$ matrices with entries in $R$ ) such that $a=\sum\left\{\mu f_{1, N} \cdot f: f \in F(X)\right\}$ (abbreviated as $\sum \mu f_{1, N} \cdot f$ ).

Proof. (1) The condition is necessary. This is trivial if $a=\pi_{1} a$. Hence it suffices to show that for any $r, r^{\prime} \in R, a=\sum \mu f_{1, N} \cdot f$ and $a^{\prime}=\sum \mu^{\prime} f_{1, N^{\prime}} \cdot f$ one can construct suitable homomorphisms giving $r a+a^{\prime} r^{\prime}, a a^{\prime}$ and $a^{*}$. This is done below, defining the homomorphisms by their restriction to $X$. 
Addition. Let $N^{\prime \prime}=N+N^{\prime}+2$ and $\mu^{\prime \prime} x \in R^{N^{\prime \prime} \times N^{\prime \prime}}$ defined for each $x \in X$ by

$$
\begin{aligned}
\mu^{\prime \prime} x_{i, 1} & =\mu^{\prime \prime} x_{N^{\prime \prime}, i}=0 \quad \text { for } 1 \leqq i \leqq N^{\prime \prime} ; \\
\mu^{\prime \prime} x_{1, i+1} & =r \mu x_{1, i} \text { and } \mu^{\prime \prime} x_{i+1, N^{\prime \prime}}=\mu x_{i, N} \quad \text { for } 1 \leqq i \leqq N ; \\
\mu^{\prime \prime} x_{1, i+N+1} & =\mu^{\prime} x_{1, i} \text { and } \mu^{\prime \prime} x_{i+N+1, N^{\prime \prime}}=\mu^{\prime} x_{i, N^{\prime}} \cdot r^{\prime} \quad \text { for } 1 \leqq i \leqq N^{\prime} ; \\
\mu^{\prime \prime} x_{i, i^{\prime}} & =\text { the direct sum of } \mu x \text { and } \mu^{\prime} x \quad \text { for } 2 \leqq i, i^{\prime} \leqq N^{\prime \prime}-1 ; \\
\mu^{\prime \prime} x_{1, N^{\prime \prime}} & =r \mu x_{1, N}+\mu^{\prime} x_{1, N^{\prime}} r^{\prime} .
\end{aligned}
$$

The verification is trivial.

Product. Let $N^{\prime \prime}=N+N^{\prime}$ and define $\nu f \in R^{N^{\prime \prime} \times N^{\prime \prime}}$ for each $f \in F(X)$ by $\nu f_{i, i^{\prime}}=\mu f_{i, N}$ if $f \neq 1,1 \leqq i \leqq N, i^{\prime}=N+1 ; \nu f_{i, i^{\prime}}=0$, otherwise. Then, if $\mu^{\prime \prime} x=\bar{\mu} x+\nu x$ where $\bar{\mu} x$ is the direct sum of $\mu x$ and $\mu^{\prime} x$, one has for each $f=x^{(1)} x^{(2)} \cdots x^{(n)}, \mu^{\prime \prime} f=\bar{\mu} f+\sum\left\{\bar{\mu} f^{\prime} \nu x^{(j)} \bar{\mu} f^{\prime \prime}: f^{\prime} x^{(j)} f^{\prime \prime}=f\right\}$. Since $\nu f x^{(j)}=\bar{\mu} f \nu x^{(j)}$ and $\left(\nu f^{\prime \prime \prime} \bar{\mu} f^{\prime \prime}\right)_{1, N^{\prime \prime}}=0$ when $f^{\prime \prime}=1$, one has $\mu^{\prime \prime} f_{1, N^{\prime \prime}}=\sum\left\{\left(\mu f_{1, N}^{\prime}\right)\left(\mu^{\prime} f_{1, N^{\prime}}^{\prime \prime}\right): f^{\prime} f^{\prime \prime}=f\right\}$. Hence, $\sum \mu^{\prime \prime} f_{1, N^{\prime \prime}} \cdot f=a a^{\prime}$.

Quasi-inverse. Let $N^{\prime \prime}=N$ and define $\nu f \in R^{N \times N}$ for each $f \in F(X)$ by $\nu f_{i, i^{\prime}}=\mu f_{i, N}$ if $f \neq 1,1 \leqq i \leqq N, i^{\prime}=1 ; \nu f_{i, i^{\prime}}=0$, otherwise. Then $\mu^{\prime \prime} x=\mu x+\nu x$ and since $\mu f \nu x=\nu f x$ identically one has $\mu^{\prime \prime} f$ $=\sum \nu f^{(1)} \nu f^{(2)} \cdots \nu f^{(k)} \mu f^{(k+1)}$ where the summation is over all the factorisations $f=f^{(1)} f^{(2)} \cdots f^{(k+1)}$ of $f$ in an arbitrary number of factors. The $(1, N)$ entry of any of these products is zero unless all its factors are different from 1 and under this condition, it is equal to $\mu f_{1, N}^{(1)} \mu f_{1, N}^{(2)} \cdots \mu f_{1, N}^{(k+1)}$. Hence, $\sum \mu^{\prime \prime} f_{1, N} \cdot f=\sum_{n>0} a^{n}=a^{*}$ and the first part of the proof is completed.

(2) The condition is sufficient. We say that the proper system $p$ is linear if for each $j \leqq M, p_{j}=q_{j, 0}+\sum_{j^{\prime}} q_{j, j^{\prime}} y_{j^{\prime}}$, where all the $q$ 's belong to $R_{\mathrm{rat}}^{*}(X)$ and we verify that all coordinates of the solution of such a system belong to $R_{\text {rat }}^{*}(X)$.

This is trivial if $M=1$ because $p(\infty)=\left(1-q_{1,1}\right)^{-1} q_{1,0}\left(=\left(1+q_{1,1}^{*}\right) q_{1,0}\right)$. If it is true for $M^{\prime}<M$ it is still true for $M$. Indeed, because $p(\infty)_{M}$ $=\left(1-q_{M, M}\right)^{-1}\left(q_{M, 0}+\sum_{j<M} q_{M, j^{\prime}} p(\infty)_{j^{\prime}}\right)$, the proper linear system $p^{\prime}$ defined by $p_{j}^{\prime}=p_{j}-q_{j, M} y_{M}+q_{j, M} p_{M}$ for $j<M$ and $p_{M}^{\prime}$ $=\left(1-q_{M, M}\right)^{-1}\left(p_{M}-q_{M, M} y_{M}\right)$ is such that $p(\infty)=p^{\prime}(\infty)$. Since its first $M-1$ coordinates do not involve $y_{M}$ the result follows from the induction hypothesis.

Now, given a homomorphism $\mu$ of $F(X)$ into $R^{M \times M}$, the $M$ elements $a_{j}=\sum\left\{\mu f_{j, M} \cdot f: f \in F(X), f \neq 1\right\}$ are such that $\left(a_{j}, x f\right)$ $=\sum_{j^{\prime}} \mu x_{j, j^{\prime}}\left(a_{j^{\prime}}, f\right)$. Hence $\left(a_{1}, \cdots, a_{M}\right)$ is the solution of the linear proper system such that $q_{j, 0}=\sum\left\{\mu x_{j, M} \cdot x: x \in X\right\}, \quad q_{j, j^{\prime}}$ $=\sum\left\{\mu x_{j^{\prime}, j} \cdot x: x \in X\right\}$ for each $j, j^{\prime}$ and 2.1 is proved. 
We now consider two subrings $R^{\prime}$ and $R^{\prime \prime}$ of $R$ that commute element-wise.

Property 2.2. If $a=\sum \mu^{\prime} f_{1, N} \cdot f \in R_{\mathrm{rat}}^{\prime *}(X)$ where $\mu^{\prime}$ is a homomorphism into $R^{\prime N \times N}$ and if $b=p(\infty)_{1} \in R_{\text {alg }}^{\prime \prime *}(X)$ where the proper system $p$ has its coordinates in $R_{\mathrm{pol}}^{\prime \prime *}(X \cup Y)$, then $a \odot b \in R_{\mathrm{alg}}^{*}(X)$. If, further, $b \in R_{\mathrm{rat}}^{\prime \prime *}(X)$ then $a \odot b \in R_{\mathrm{rat}}^{*}(X)$.

Proof. We verify first the case of $b \in R_{\text {rat }}^{\prime \prime *}(X)$, i.e., of $b=\sum \mu^{\prime \prime} f_{1, N^{\prime \prime}} \cdot f$ for some $N^{\prime \prime}$ and $\mu^{\prime \prime}$. Then $a \odot b=\sum\left(\mu^{\prime} \otimes \mu^{\prime \prime}\right) f_{1, N N^{\prime}} \cdot f$ where the kroneckerian product $\mu^{\prime} \otimes \mu^{\prime \prime}$ is a homomorphism of $F(X)$ into $R^{N N^{\prime \prime} \times N N^{\prime \prime}}$ because $R^{\prime}$ and $R^{\prime \prime}$ commute and the result is proved.

For the general case we denote by $K(Z)$ for any set $Z$ the ring of the $N \times N$ matrices with entries in $R(Z)$. We shall have to consider several homomorphisms of module $\sigma: R^{M}\left(Z^{\prime}\right) \rightarrow K^{M}\left(Z^{\prime \prime}\right)$ where $Z^{\prime}$ and $Z^{\prime \prime}$ are two finite sets. In each case $\sigma$ is defined by a mapping $Z^{\prime} \rightarrow K\left(Z^{\prime \prime}\right)$ which is extended in a natural fashion to a homomorphism of the monoid $F\left(Z^{\prime}\right)$ into the multiplicative structure of $K\left(Z^{\prime \prime}\right)$. Then for each

$$
a=\left(a_{1}, \cdots, a_{M}\right) \in R^{M}\left(Z^{\prime}\right), \quad \sigma a_{j}=\sum\left\{\left(a_{j}, g\right) \cdot \sigma g: g \in F\left(Z^{\prime}\right)\right\}
$$

and $\sigma a=\left(\sigma a_{1}, \cdots, \sigma a_{M}\right)$.

More specifically, $\mu: R^{M}(X) \rightarrow K^{M}(X)$ is induced by a mapping $\mu: X \rightarrow K(X)$ such that the entries of each $\mu x$ belong to $R^{* *}(X)$.

For each $q \in R^{\prime \prime * M}(X), \lambda_{\mu q}: R(X \cup Y) \rightarrow K^{M}(X)$ is induced by $\lambda_{\mu q} f$ $=\mu f$ if $f \in F(X)$ and $\lambda_{\mu q} y_{j}=\mu q_{j}$ if $y_{j} \in Y$. Hence, since $R^{\prime}$ and $R^{\prime \prime}$ commute element-wise, $\mu \lambda_{q} g=\lambda_{\mu q} g$ for each $g \in F\left(X \cup Y\right.$ ) (with $\lambda_{q}$ as previously defined). Consequently, $\mu \lambda_{q} p=\lambda_{\mu q} p$ for any $p \in R^{\prime \prime M}(X \cup Y)$.

Let now $Z=\left\{z_{j, i, i^{\prime}}\right\}\left(1 \leqq j \leqq M ; 1 \leqq i, i^{\prime} \leqq N\right)$, a set of $M \times N \times N$ new variables and $\nu: R^{M}(X \cup Y) \rightarrow K^{M}(X \cup Z)$ induced by $\nu f=\mu f$ if $f \in F(X), \nu y_{j}=$ the $N \times N$ matrix with entries $z_{j, i, i^{\prime}}$ if $y_{j} \in Y$. Also $\lambda_{\nu q}: R(X \cup Z) \rightarrow R(X)$ is induced by $\lambda_{v q} f=f$ if $f \in F(X)$ and $\lambda_{v q} z_{j, i, i^{\prime}}$ $=\left(\nu q_{j}\right)_{i, i^{\prime}}$ if $z_{j, i, i^{\prime}} \in Z$. We extend $\lambda_{\nu q}$ to a homomorphism $K^{M}(X \cup Z)$ $\rightarrow K^{M}(X)$ by defining $\lambda_{\nu q} m$ for any $m \in K(X \cup Z)$ as the $N \times N$ matrix with entries $\lambda_{v q}\left(m_{i, i^{\prime}}\right)$.

Because $R^{\prime}$ and $R^{\prime \prime}$ commute, $\lambda_{\mu q} g=\lambda_{\nu q} \nu g$ for each $g \in F(X \cup Y)$ and, consequently, $\lambda_{\mu q} p=\lambda_{\nu q} \nu p$ for each $p \in R^{\prime \prime * M}(X \cup Y)$. Hence, if $p$ is a proper $M$-dimensional system with coordinates in $R^{\prime \prime *}(X \cup Y)$ we have $\mu p(\infty)=\mu \lambda_{p(\infty)} p=\lambda_{\mu p(\infty)} p$. Since $\mu$ and $\nu$ coincide on $R^{\prime \prime * M}(X)$, we have also $\mu p(\infty)=\nu p(\infty)=\lambda_{\mu p(\infty)} p=\lambda_{\nu p(\infty)} \nu p$.

However, the $M \times N \times N$ elements $p_{j, i, i^{\prime}}^{\prime}=\left(\nu p_{j}\right)_{i, i^{\prime}}$ all belong to $R^{*}(X \cup Z)$ and they constitute a proper system $p^{\prime}$ of dimension $M N^{2}$. Thus, by construction, $\left(\mu p(\infty)_{j}\right)_{i, i^{\prime}}=p^{\prime}(\infty)_{j, i, i^{\prime}}$ identically. If, fur- 
ther, $p \in R_{\text {pol }}^{\prime \prime *}(X \cup Y)$ all the entries appearing in $\nu p$ belong to $R_{\mathrm{pol}}^{*}(X \cup Z)$ and then finally $\left(\mu p(\infty)_{j}\right)_{i, i^{\prime}} \in R_{\mathrm{alg}}^{*}(X)$.

This completes the proof because

$$
\begin{aligned}
a \odot b & =\sum\left\{(b, f) \mu^{\prime} f_{1, N} \cdot f: f \in F(X)\right\} \\
& =\sum\left\{(b, f) \mu f_{1, N}: f \in F(X)\right\}=\mu b_{1, N}
\end{aligned}
$$

where for each $x \in X, \mu$ is defined by $\mu x_{i, i^{\prime}}=\mu^{\prime} x_{i, i^{\prime}} \cdot x$.

REMARK 1. Definitions 1,2 , and 3 and the computations of this section used only the structure of monoid of the additive groups considered. Hence, the results are still valid when an arbitrary semiring $S$ is taken in place of $R$. For $S$ consisting of two Boolean elements, Jungen's theorem and its special case for $b$ rational have been obtained in a different form by Y. Bar-Hillel, M. Perles and E. Shamir [1] (also by S. Ginsburg and G. F. Rose [5]) and by S. Kleene [8] respectively as by-products of more sophisticated theories.

REMARK 2. Let $R=C$, the field of complex numbers; and $p$ a proper system of dimension $M$. Introducing $4 M$ new symbols $z_{j}$ and replacing each $y_{j}$ by $z_{4 j}+i z_{4 j+1}-z_{4 j+2}-i z_{4 j+3}$ in the $p_{j}$ s we can deduce from $p$ a new system of dimension $4 M$ in which all the coefficients are non-negative real numbers and whose solution is simply related to $p(\infty)$.

Assume now that $p \in C_{\mathrm{pol}}^{* M}(X \cup Y)$ has only real non-negative coefficients and denote by $\alpha$ a homomorphism of $C_{\mathrm{pol}}(X \cup Y)$ into $C$. Because of the assumption that $\left(p_{j}, y_{j^{\prime}}\right)=\left(p_{j}, 1\right)=0$, identically, we can find an $\epsilon>0$ such that $\left|\alpha p_{j}\right|<\epsilon$ for all $j$ when $|\alpha x| \leqq \epsilon$ and $|\alpha y| \leqq 2 \epsilon$ for all $x \in X$ and $y \in Y$. Since the sequence $\alpha p(0), \alpha p(1)$, $\cdots, \alpha p(n), \cdots$ is monotonically increasing it converges to a finite solution (cf., e.g., [10]).

Hence, the canonical epimorphism of $C_{\mathrm{pol}}(X \cup Y)$ onto the ring of the ordinary (commutative) polynomials can be extended to an epimorphism of $C_{\mathrm{alg}}(X)$ onto the ring of the Taylor series of the algebraic functions.

Acknowledgment. Acknowledgment is made to the Commonwealth Fund for the grant in support of the visiting professorship of biomathematics in the Department of Preventive Medicine at Harvard Medical School.

\section{REFERENCES}

1. Y. Bar-Hillel, M. Perles and E. Shamir, On formal properties of simple phrase structure grammars, Technical Report No. 4. Information System Branch, Office of Naval Research, 1960. 
2. S. Bochner and W. T. Martin, Singularities of composite functions in several variables, Ann. of Math. 38 (1938), 293-302.

3. R. H. Cameron and W. T. Martin, Analytic continuation of diagonals, Trans. Amer. Math. Soc. 44 (1938), 1-7.

4. K. T. Chen, R. H. Fox and R. C. Lyndon, Free differential calculus. IV, Ann. of Math. (2) 68 (1958), 81-95.

5. S. Ginsburg and G. F. Rose, Operations which preserve definability, System Development Corporation, Santa Monica, Calif., SP-511, October, 1961.

6. U. S. Haslam-Jones, An extension of Hadamard multiplication theorem, Proc. London Math. Soc. II. Ser. 27 (1928), 223-232.

7. R. Jungen, Sur les series de Taylor n'ayant que des singularités algébrico-logarithmiques sur leur cercle de convergence, Comment. Math. Helv. 3 (1931), 226-306.

8. S. Kleene, Representation of events in nerve nets and finite automata, Automata Studies, Princeton Univ. Press, Princeton, N. J., 1956.

9. M. Lazard, Lois de groupes et analyseurs, Ann. Sci. Ecole Norm. Sup. (4) 72 (1955), 299-400.

10. A. M. Ostrowski, Solutions of equations and systems of equations, Academic Press, New York, 1960.

Harvard Medical School 